\title{
A Structurally Variable Hinged Tetrahedron Framework from DNA Origami
}

\author{
David M. Smith, ${ }^{1,2}$ Verena Schüller, ${ }^{1}$ Carsten Forthmann, ${ }^{3,4}$ Robert Schreiber, ${ }^{1}$ \\ Philip Tinnefeld, ${ }^{2,4}$ and Tim Liedl ${ }^{1,2}$ \\ ${ }^{1}$ Physik Weicher Materie und Biophysik, Ludwig Maximilian University, 80539 Munich, Germany
${ }^{2}$ Center for NanoScience, Ludwig Maximilian University, 80799 Munich, Germany
${ }^{3}$ Angewandte Physik-Biophysik, Ludwig Maximilian University, 80539 Munich, Germany
${ }^{4}$ Physikalische und Theoretische Chemie-NanoBioScience, Braunschweig University of Technology, 38106 Braunschweig, Germany
}

Correspondence should be addressed to David M. Smith, david.smith@physik.uni-muenchen.de

Received 15 May 2011; Accepted 27 June 2011

Academic Editor: F. C. Simmel

Copyright ( $) 2011$ David M. Smith et al. This is an open access article distributed under the Creative Commons Attribution License, which permits unrestricted use, distribution, and reproduction in any medium, provided the original work is properly cited.

\begin{abstract}
Nanometer-sized polyhedral wire-frame objects hold a wide range of potential applications both as structural scaffolds as well as a basis for synthetic nanocontainers. The utilization of DNA as basic building blocks for such structures allows the exploitation of bottom-up self-assembly in order to achieve molecular programmability through the pairing of complementary bases. In this work, we report on a hollow but rigid tetrahedron framework of $75 \mathrm{~nm}$ strut length constructed with the DNA origami method. Flexible hinges at each of their four joints provide a means for structural variability of the object. Through the opening of gaps along the struts, four variants can be created as confirmed by both gel electrophoresis and direct imaging techniques. The intrinsic site addressability provided by this technique allows the unique targeted attachment of dye and/or linker molecules at any point on the structure's surface, which we prove through the superresolution fluorescence microscopy technique DNA PAINT.
\end{abstract}

\section{Introduction}

The design and self-assembly of DNA strands into precisely defined objects on the nanometer scale has emerged as a promising technique in the field of nanotechnology. Stemming from the initial idea of generating periodic lattices from DNA [1], the concurrent exploitation of (i) complimentary base pairing between short strands, (ii) branch-like Holliday junctions, and (iii) the inherent helical twist of doublestranded DNA complexes has allowed for the assembly of small, identical motifs which constitute the repeating unit cells of periodic two-dimensional sheets or threedimensional crystal structures extending nearly to the millimeter scale [2-5]. The development of techniques to build rigid, three-dimensional DNA-based structures is, however, an important aspect to the future utilization of this methodology in nanofabrication [6-11]. While many attempts to construct simple three-dimensional polyhedra have been fraught with problems of instability, unwanted by-products, low-yield, or overly complex synthesis strategies, the recent utilization of hierarchical assembly schemes [12] and the DNA origami technique $[13,14]$ has provided a path towards the relatively simple generation of uniform populations.

The DNA origami technique is based on the use of a long, usually circular "scaffold" strand, which is folded and clamped into a desired shape by hybridization with hundreds of shorter "staple" oligonucleotides [13]. In contrast to earlier schemes for generating nanostructures from synthesized oligonucleotides, the utilization of a viral scaffold, which is typically the $7 \mathrm{~kb}$ circular single-stranded M13mp18 bacteriophage genome or alternatively PCR-templated products $[15,16]$, allows for the construction of far larger structures often extending to several hundreds of nanometers [17]. The heterogeneous sequence of the underlying scaffold strand forms the basis for the unique strength of this method, by which each staple strand has the potential to act as a "handle" for the placement of accessory molecules at virtually any site in the structure with nanometer precision 
Journal of Nucleic Acids

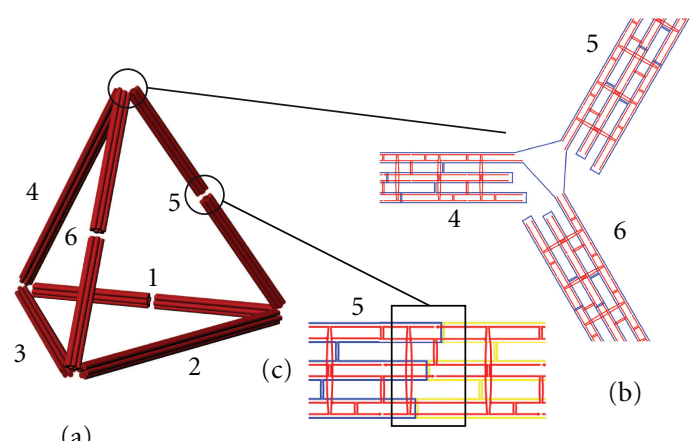

Figure 1: Schematic view of DNA origami tetrahedron. (a) In the three-dimensional representation, each double-helical section is represented by a single red cylinder. The six struts are labeled 1 through 6 , and each consist of a bundle of six parallelly connected double helices. (b) A two-dimensional diagram of the hinged vertex formed by struts 4, 5, and 6 shows the path of the m13MP18-based scaffold (blue) forming the single-stranded connections between bundles and the pattern of staple oligonucleotides (red) around the hinge. (c) Local structure of the scaffold path within strut 5 shows three closely aligned scaffold crossovers stabilized by short staple sections forming the weakened gap. The scaffold path on opposite sides of the gap is marked in either yellow or blue to emphasize the discontinuity.

$[18,19]$. Through standard purification techniques, relatively uniform populations of folded structures of the desired conformation can be isolated. This method is suited not only for compact, structurally rigid objects, but also for those which incorporate single-stranded sections amongst rigidly extended helical bundles to generate objects displaying flexible hinges and inherent tensile strain [20].

Hollow, load-bearing frameworks represent one class of structures which holds a broad relevance in applied nanoscience as potential container systems. The use of programmed DNA self-assembly offers a highly suitable alternative pathway in their construction, as has been demonstrated in several recent studies. Two in particular have succeeded in generating highly uniform populations of rigid nanostructures. Small tetrahedral structures measuring less than 10 nanometers per strut have been rapidly assembled from four distinct oligonucleotides and have shown a significant mechanical stability, withstanding compression forces in excess of $100 \mathrm{pN}$ before exhibiting buckling [10]. It was later demonstrated that a hierarchical self-assembly concept utilizing simple repeating motifs from a relatively small number of oligonucleotides [12] or DNA origami-based subunits [14] can be used to generate larger polyhedra suitable as containers or for the orientation of functional units in space with nanometer precision. While the above strategies based upon hybridization of shorter oligonucleotides produce high yields of correctly assembled products, the former is significantly size-limited based on its dependence on a simple annealing scheme, while the ability to selectively attach molecules at distinct points on the polyhedra constructed by hierarchical means suffers due to the redundancy of strands or units within the repeated motifs. The use of subunits constructed from DNA origami [14] can solve this issue of redundancy via a simple internal shifting of the scaffold strand; however, it does require the additional and often intricate control over correct threedimensional orientation and self-assembly of the multiple segments. Consequently, the fabrication of enclosed hollow frames from a single DNA scaffold via the origami method, as previously demonstrated in a spherical architecture [21], offers the potential for the straightforward generation of a wide variety of fully and uniquely addressable structures.

As shown in the present study, utilization of the DNA origami method to construct a rigid, enclosed tetrahedral framework, illustrated in Figure 1, provides the ability to generate structures reaching a size of 75 nanometers along each edge while retaining the full addressability afforded by the heterogeneous sequence of the scaffold strand. Single stranded "hinges" at each of the four vertices of the tetrahedron allow for a structural variability. By selective staple oligonucleotide omission, four unique two-dimensional structures varying in length and width between 75 nanometers and 300 nanometers are generated. Even though the full three-dimensional structure displays mechanical rupture likely due to electrostatic-mediated surface binding and flow-induced shearing, proper annealing can nevertheless be confirmed through gel electrophoresis and examination of the assorted open frames. Additionally, binding of fluorescent molecules at precisely determined points on the structure emphasizes its potential to act as a threedimensional ruler for emerging superresolution microscopy methods such as DNA-PAINT and Blink [22-24].

\section{Materials and Methods}

2.1. Preparation of DNA Structures. Reverse-phase cartridgepurified unmodified staple oligonucleotides were shipped dry from Bioneer Corporation (Alameda, Calif, USA) and resuspended to a concentration of $100 \mu \mathrm{M}$ in $\mathrm{H}_{2} \mathrm{O}$. HPLC-purified dye- and biotin-modified oligonucleotides for PAINT analysis were purchased from IBA $\mathrm{GmbH}$ (Göttingen, Germany) at a concentration of $50 \mu \mathrm{M}$ in $\mathrm{H}_{2} \mathrm{O}$. The ATTO655-labeled oligonucleotide contained the sequence $5^{\prime}$-GGT GAA GA $A_{\text {ATTO655 }}-3^{\prime}$ and the biotinmodified strand $5^{\prime}$-GGT AGT AAT AGG AGA ATG $\mathrm{bt}_{\mathrm{t}}-3^{\prime}$. The 8634-nucleotide-long M13mp18-phage-based scaffold strand was prepared and isolated as previously described [14]. 
As a general annealing condition, $10 \mathrm{nM}$ of scaffold strand and $100 \mathrm{nM}$ of each staple were mixed in TE buffer $\left(10 \mathrm{mM}\right.$ Tris- $\mathrm{HCl}+1 \mathrm{mM}$ EDTA, $\mathrm{pH} 8.0$ at $\left.20^{\circ} \mathrm{C}\right)$ containing $14 \mathrm{mM} \mathrm{MgCl}$, heated to $80^{\circ} \mathrm{C}$ for 5 minutes, and quickly cooled to $60^{\circ} \mathrm{C}$ over 20 minutes, before being slowly cooled to $24^{\circ} \mathrm{C}$ in steps of $0.5^{\circ} \mathrm{C}$ over approximately 40 hours. When applicable for dye or biotin attachment, staple oligonucleotides with docking handle extensions replaced unextended staples in the solution and biotin- or dyemodified oligonucleotides were included at $500 \mathrm{nM}$ per staple during annealing. For PAINT analysis, a total of six biotin attachment sites (two per vertex) and three ATTO655 sites (one per strut) were used to modify struts I, II, and III, as shown in Figure 4(a) and in the Supplementary Materials in Figure S1.

Gel electrophoresis was performed with an agarose concentration of either $2.0 \%$ (for band analysis) or $0.7 \%$ (for structure purification). Agarose was first heated to boiling temperature in $45 \mathrm{mM}$ Tris borate $+1 \mathrm{mM}$ EDTA ( $\mathrm{pH} 8.2$ at $20^{\circ} \mathrm{C}$ ) then cooled to $60^{\circ} \mathrm{C}$ in an ice bath, at which point $\mathrm{MgCl}_{2}$ was added to a final concentration of $11 \mathrm{mM}$, before being cooled to room temperature in a gel cask. For sample preparation, $5 \mu \mathrm{L}$ of DNA origami structures were mixed with $3 \mu \mathrm{L}$ of $6 \mathrm{x}$ agarose gel loading buffer (30\% glycerol weight-to-volume in water, $0.025 \%$ xylene cyanol, $0.025 \%$ bromophenol blue) and brought to a total volume of $18 \mu \mathrm{L}$ in $\mathrm{H}_{2} \mathrm{O}$, for roughly $5 \cdot 10^{-14}$ moles of structures per gel pocket. Similarly, bare scaffold strands were mixed with loading buffer and $\mathrm{H}_{2} \mathrm{O}$ to give a total amount of $1 \cdot 10^{-13}$ moles per sample. The different gel pockets were filled with the origami and scaffold samples along with a $1 \mathrm{~kb}$ DNA ladder and run for approximately 3 hours at constant $70 \mathrm{~V}$ over a cathode-anode distance of $22 \mathrm{~cm}$. The entire apparatus was cooled in an ice water bath while running to avoid unwanted structure denaturation. Afterwards, the gel was stained with an ethidium bromide solution at $0.5 \mu \mathrm{g} / \mathrm{mL}$ and imaged under $302 \mathrm{~nm}$ UV excitation. Following electrophoresis, the primary band was excised from the gel and centrifuged at $5000 \times \mathrm{g}$ for 7 minutes in spin columns (Freeze'n Squeeze Spin Columns, Biorad) to isolate structures. Purified origami samples were usually estimated to contain approximately $1 \mathrm{nM}$ of annealed structures.

\subsection{Visualization of Structures (TEM and AFM)}

TEM Imaging. Formvar-supported carbon coated TEM grids were purchased from Plano $\mathrm{GmbH}$ (Wetzlar, Germany). Grids were first hydrophilicized in a Diener Electronic Femto plasma cleaner. $2 \mu \mathrm{L}$ of purified origami samples was added to the grids, and structures were allowed to bind for 60 seconds. Excess sample was then quickly removed from the grids by absorption with filter paper. Grids were quickly washed with $1 \%$ uranyl acetate in $\mathrm{H}_{2} \mathrm{O}$, then stained for 15 seconds with the same and allowed to dry completely. The samples were then imaged with a JEM-1011 transmission electron microscope (JEOL) operated at $100 \mathrm{kV}$.

AFM Imaging. For viewing with atomic force microscopy, $5 \mu \mathrm{L}$ of purified origami sample placed onto a freshly cleaved mica surface (Plano GmbH, Wetzlar, Germany) which had been attached by hot glue to a $15 \mathrm{~mm}$ metal specimen disc (Ted Pella, Inc., Redding, Calif, USA). Structures were allowed to bind for 60 seconds before being washed twice with $30 \mu \mathrm{L}$ of TE buffer solution containing $12.5 \mathrm{mM} \mathrm{MgCl}_{2}$ to remove unbound origami objects and other debris. Samples were imaged in Tapping Mode in the previously mentioned TE/ $\mathrm{Mg}^{++}$buffer conditions using a NanoScope III Multimode AFM from Digital Instruments (Veeco Instruments Ltd., Plainview, Tex, USA) with a silicon-nitride tip with a spring constant of $k=0.24 \mathrm{~N} / \mathrm{m}$ (Veeco).

\subsection{Shape Analysis by DNA PAINT}

Sample Preparation. Chambered Cover Glass Slides (LabTek, NUNC, Langenselbold, Germany) were prepared for Total Internal Reflection (TIR) Microscopy in the following manner: glass surfaces were first cleaned with $0.1 \mathrm{M} \mathrm{HF}$ for 30 seconds and washed with PBS. They were then passivated with a mixture of $5 \mathrm{mg} / \mathrm{mL}$ bovine serum albumin (BSA) and $1 \mathrm{mg} / \mathrm{mL}$ biotin-conjugated BSA for 16 hours. Following a second washing with PBS to remove unbound proteins, the surface was incubated for 15 minutes with $0.01 \mathrm{mg} / \mathrm{mL}$ streptavidin (IBA $\mathrm{GmbH}$ ) then again washed with PBS to remove any excess, unbound streptavidin. $50 \mu \mathrm{L}$ of the sample solution which contained $90 \%$ of a $1 \mathrm{M}$ $\mathrm{MgCl}_{2}$ buffer and $10 \%$ purified origami solution (for a final structure concentration of approximately $0.1 \mathrm{nM}$ ) was added to the chamber and allowed to bind to the surface. Binding was facilitated by biotin linkers on the origami structures, included at each vertex of the plane formed by struts I, II, and III. Chambers were again washed with PBS to remove unbound structures, and a $50 \mathrm{nM}$ solution of ATTO655labeled oligonucleotides (PAINT-DNA) in $500 \mathrm{mM} \mathrm{NaCl}$ was added.

Microscopy Setup. An inverted Olympus IX-71 in objectivetype TIRF configuration outfitted with a UPlanSApo objective (100x, NA 1.40, oil immersion) was used for monitoring. Dyes were excited by a single-mode diode laser (XTL, Toptica Photonics, Grafelfing, Germany) operated at $100 \mathrm{~mW}$ with a wavelength of $\lambda=650 \mathrm{~nm}$. Fluorescence was imaged on an EMCCD camera (Ixon DU-897, Andor Technologies, Belfast, Northern Ireland). Image size was $128 \times 128$ pixels, with each pixel representing a length of approximately $90 \mathrm{~nm}$. Each acquired sequence of images contained approximately 2000 frames taken at a rate of $20 \mathrm{~Hz}$.

\section{Results and Discussion}

3.1. Tetrahedron Design. The tetrahedral design was implemented using the caDNAno [25] software package, which was developed to facilitate the layout of both three-dimensional and flat DNA origami structures. A circular scaffold strand of 8634 nucleotides was used to allow for a maximal size, which was folded into its final configuration by a total of 211 staple strands. Each of the six struts in the tetrahedron is based on a bundle of six parallel double helices [14, 26], 227 paired bases in length, 
with each double helix represented by a cylinder in the three-dimensional schematic (Figure 1(a)). The bundles are connected at each of the four hinged vertices to the two neighboring bundles by a four-base single-stranded section of the circular scaffold extending between their termini, as indicated in the inset in Figure 1(b). At each vertex, adjacent double helices within a single six-helix bundle were selected as connecting points to each of the two neighboring struts so as to minimize possible strains resulting from sterically induced stretching of the roughly $4 \mathrm{~nm}$ single-stranded connecting section. Additionally, connection points on opposite ends of a single strut extend from the same pair of double-stranded helical sections, which minimizes any possible unfavorable twist-strain within the bundle $[21,27]$. We have adopted the nomenclature as indicated in Figure 1(a) whereby each strut is labeled 1 through 6. Due to circular continuity of the scaffold strand and the periodicity of scaffold crossovers between neighboring helices within a single six-helix bundle, struts 1,5 , and 6 contain points where three scaffold crossovers are contained within a seven-base section along the double helix. As highlighted in Figure 1(c) for strut 5, the three nearly aligned gaps within the strut are stabilized only by staple oligonucleotides hybridized to scaffold sections on opposing sides of each opening. Lacking a direct connection from the internal scaffold path running throughout the structure, these junctions, referred to as 1,5 , and 6 in coordination with the previous nomenclature, must be viewed as weak points susceptible to rupture; however, they provide the overall tetrahedron with its structural variability as will be discussed in later sections. A full schematic of both scaffold and staple arrangement within the overall structure can be seen in the Supplementary Materials in Figure S1.

Specific binding of tetrahedra to surfaces was facilitated by the addition of an 18-base sequence on the $3^{\prime}$ end of six staples, each terminating at the end of a bundle lying on the plane formed by struts 1,2, and 3, to which a complementary oligonucleotide containing a biotin molecule conjugated to its $3^{\prime}$ end could be hybridized. This allowed stable attachment of structures to surfaces coated with biotin-binding proteins such as streptavidin. The positioning of the biotin on the $3^{\prime}$ end of the modified strand directly abutting the structure imposes the closest possible binding between the object and surface. In a similar manner, one staple positioned along the length of each of struts 1,2 , and 3 with their $3^{\prime}$ terminus on the outer surface of the bundle was selected as a handle for complimentary oligonucleotides with an ATTO655 dye conjugated to the $3^{\prime}$ end of the strand in DNA PAINT experiments. In both cases, any combination of biotin or dye sites could be included within the structure by the replacement of normal staple strands with those extended by the complimentary "handle" or docking sequence.

3.2. Full Tetrahedron Structural Properties. The structures were annealed in a one-pot reaction in TE buffer in the presence of $14 \mathrm{mM} \mathrm{MgCl}_{2}$. As analyzed by agarose gel electrophoresis after annealing, well-defined bands were observed indicating a population of uniformly annealed structures, along with signs of additional slower migrating aggregate products, as can be seen in the Supplementary Materials in Figure S2. Little change in band structure was observed by varying the annealing time from 18 to 170 hours, although a lengthening of the single-stranded scaffold sections between struts caused a higher migration speed and a less-defined band structure (see Figure S2 in the Supplementary Materials). From analysis of relative band intensity in gels, the yield of primary annealed product was approximately $20 \%$. The majority of scaffold-containing material remained in the gel pockets, where presumably aggregated structures are unable to effectively enter or migrate through the gel matrix.

Annealed objects were negatively stained with uranyl acetate and analyzed via transmission electron microscopy (TEM) for structural characteristics. Despite the defined structure of gel bands, TEM observations yielded an absence of intact tetrahedra, with a large portion of the structures displaying apparent breaks along one or more of the individual struts. Two such examples are shown in the left panel of Figure 2; the individual six-helix bundle struts appear to be well formed and connectivity within most vertices is maintained; however, the structures display a deformed or flattened configuration appearing to arise from a small number of ruptures along the length of one or two edges. Measured lengths of individual struts varied from 70 to $90 \mathrm{~nm}$, close to the estimated value of $75 \mathrm{~nm}$ based on a $0.34 \mathrm{~nm}$ axial pitch between adjacent bases in the double helix.

3.3. Piecewise Verification of Proper Annealing. The pervasive existence of malformed structures under TEM observation points to one of two possible causes; either a systematic error in annealing occurs due to unexpected internal strains or kinetic traps, or properly annealed tetrahedra are denatured as a consequence of mechanical stresses endured in the process of purifying, fixing, and staining the objects. The lack of a clear predominance of any single "broken" tetrahedron configuration as viewed under TEM despite well-defined band structure in electrophoresis strongly indicates that the second consideration is the more likely explanation for the aforementioned observations. As previously emphasized, the three gap areas within each of struts 1,5 , and 6 formed by the close alignment of scaffold crossovers must be considered as weakened points along the bundles. A closer examination of the underlying staple connections across the gaps as pictured in Figure 1(c) for strut 5 shows that the hybridized sections stabilizing gaps along a single helical axis are in several cases as short as two nucleotides in overlap before the termination of the particular staple strand. While some degree of stabilization stemming from stacking interactions could occur for some abutting helices $[28,29]$, these points are nevertheless more susceptible to rupture under strong shear or compression forces than other points along continuous sections of a strut or at vertices. This can be understood through a simple comparison of the two different bond types responsible for stabilizing scaffolded DNA structures: hydrogen bonding of complimentary base pairing and the covalently connected polymer backbone comprising the main DNA scaffold through the structure. 


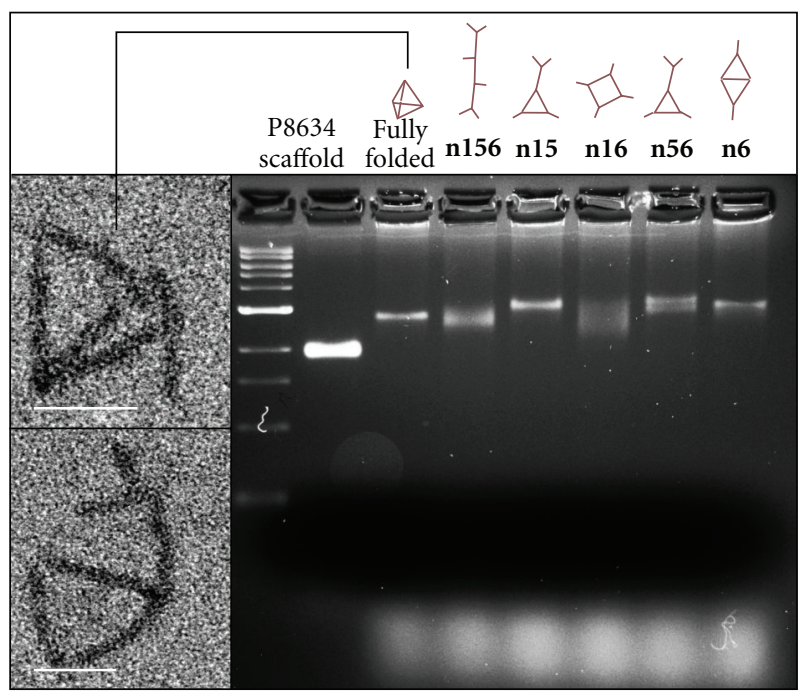

FIgure 2: (Left) Transmission electron microscopy (TEM) imaging of tetrahedron structure containing all staple nucleotides. Typically, tetrahedrons displayed one or more ruptured struts, generating the widely observed flattened configuration. Scale bars: $60 \mathrm{~nm}$. (Right) Agarose gel electrophoresis separation of the different configurations formed by targeted gap staple omission in struts 1, 5, and 6. Left to right: lanes 1-2 contain a $1 \mathrm{~kb}$ ladder and the circular p8634 scaffold, respectively. Lanes 3-8 contain the full tetrahedron, and the open configurations $\mathrm{n} 156, \mathrm{n} 15, \mathrm{n} 16, \mathrm{n} 56$, and $\mathrm{n6}$, as indicated by the sketched representations above each lane.

In the case of the former, it has been demonstrated that forces on the order of 50 piconewtons are sufficient to induce shear-oriented rupture of hybridized DNA strands [30, 31]. Conversely, earlier work has shown that covalent bonds can generally withstand forces up to the nanonewton scale before experiencing rupture [32]. Concerning the overall stability of the tetrahedron presented in this work, the relatively weak hybridized staple oligonucleotides spanning the gap regions in struts 1,5 , and 6 would be far more susceptible to rupture under external stress than any of the other areas whose stabilities are in addition to base pairing supported by the continuous, covalently-linked scaffold backbone.

Standard techniques for examining DNA-based nanostructures such as TEM or AFM depend on a strong electrostatic binding of the negatively charged structures to layers such as mica or the carbon coating on grids used for TEM, and staining protocols include washing and drying steps with rapid, high-shear addition and removal of fluids. While this has proven to cause only nominal damage to more compactly rigid structures, the weakened struts are particularly susceptible to rupture due to electrostaticdriven compression or deforming forces containing a shear component and are likely candidates for the observed open configurations compressed on the surface.

With force-induced rupturing of weakened points formed by gaps within the struts identified as a likely candidate for the observed deformation of tetrahedra, we sought to investigate the various configurations resulting from the targeted opening of those gaps in different combinations. Due to their design and the local stapling paths around these junctions, these two-dimensional conformations could be generated by the omission of staple groups spanning each of the three gaps during annealing. For this purpose, an open configuration formed by a particular combination of omitted gap staples is referred to by the number assigned to the gap or gaps presumably left open. By this nomenclature, the configuration generated by the omission of all gap staples is referred to as $\mathbf{n 1 5 6}$; that resulting from the opening of the gap along strut $\mathbf{6}$ is $\mathbf{n 6}$, and so on. While there are a total of seven possible combinations of gap staple groups which can be left out, structural redundancies mean that a total of only four possible flat configurations exist. These are roughly sketched above their corresponding lane in the gel shown in the right panel of Figure 2, with $\mathbf{n} \mathbf{6}$ being the representative structure for the three identical configurations containing a single omission.

The various flat structures were annealed and analyzed as the full tetrahedron. As can be seen in the gel in Figure 2, differences in migration and band structure amongst the variants were evident after a three-hour separation at $70 \mathrm{~V}$ over $22 \mathrm{~cm}$ in $2 \%$ agarose. Bands of the different flat two-dimensional assemblies displayed a predictably lower resolution than their fully annealed counterpart, presumably due to their increased degree of configurational flexibility around open, flexible joints. Two of these structures, the linearized $\mathrm{n} 156$ form and the rectangular $\mathrm{n} 16$, have bands with a significant population of products which migrate faster than the full tetrahedron structure containing all staples. This can be explained by the lack of structurally rigid triangular substructures within the two frames, which can lead to an elongated conformation able to effectively reptate through the gel pores. Furthermore, the geometrically redundant $\mathbf{n} 15$ and $\mathbf{n} 56$ as well as the $\mathbf{n} \mathbf{6}$ configurations all have primary populations which display a slower migration character visibly absent from the fully annealed version. In both cases, this is a strong indication that none of those are preferentially occurring in any detectible amount during annealing of the full tetrahedron and that weakened 


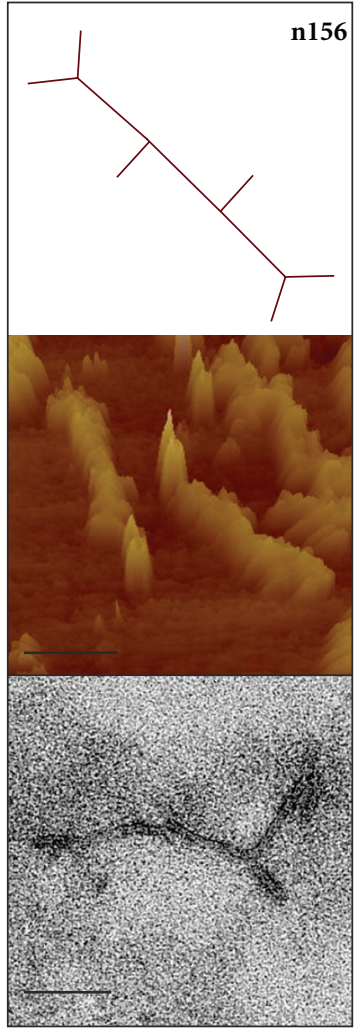

(a)

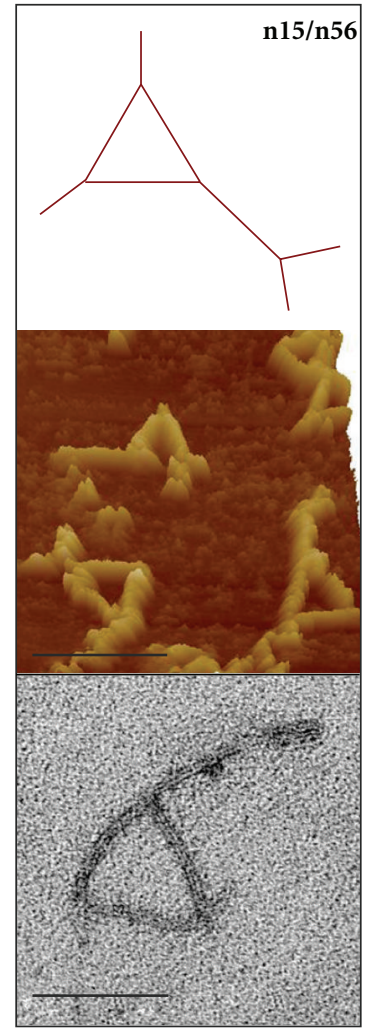

(b)

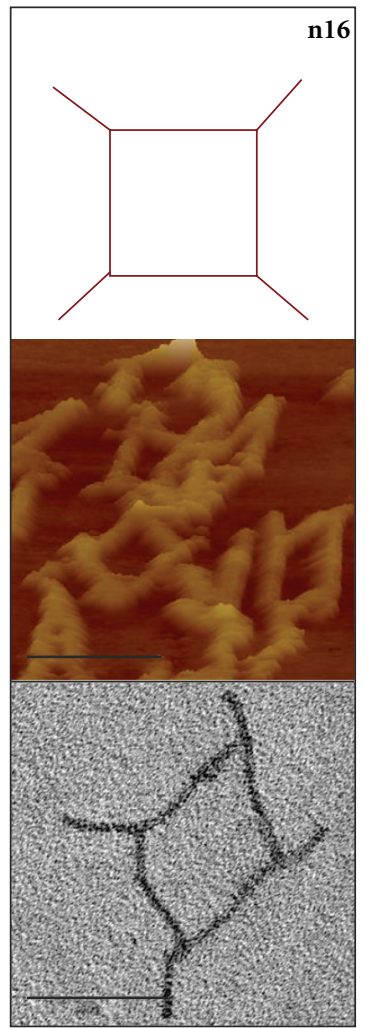

(c)

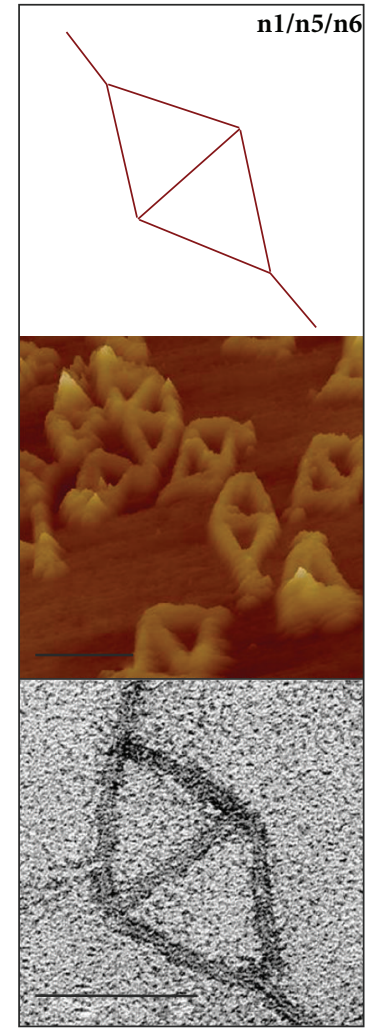

(d)

FIGURE 3: Schematic (upper row), atomic force microscopy (center), and TEM imaging (bottom) of flattened structures produced by targeted gap staple omission. (a) The chain-like $\mathbf{n} 156$ configuration is formed when all gaps are left open. (b) Removal of staple groups connecting gaps in struts 1 and 5 or alternatively 5 and 6 form the geometrically redundant $\mathbf{n} 15$ and $\mathbf{n} 56$ projections. (c) Jointed square structures n16 are formed by the opening of gaps in struts 1 and 6, displaying a degree of transverse flexibility due to possessing four flexible hinges. Omission of a single gap staple group as in (d) has a three-fold redundancy of structures with geometry indicated by the $\mathbf{n} \mathbf{6}$ object shown. Scale bars: $150 \mathrm{~nm}$ (AFM) and $75 \mathrm{~nm}$ (TEM).

points in the struts are not ruptured within the gel. This absence of any primary product resulting from the variable open configurations which displays the same migration speed as the full tetrahedron as well as the narrow band structure of the closed tetrahedron indicate that it adopts its final, defined conformation unique from those resulting from some combination of ruptured struts. Approximate yields of primary annealed products were estimated through comparison of relative band intensities. For all opened variants, this was found to be approximately $20-25 \%$, in a similar range as the fully annealed tetrahedron, although a lesser degree of band clarity does make any precise determination in this manner unreliable. Highest yields were found with the more flexible and elongated n156 and n16 variants, likely due to the greater amount of overall material able to enter the gel. Conversely, the most rigid of the variants containing only one gap opening (represented as $\mathbf{n} 6$ in Figure 2) showed the smallest yields of all products, usually under $20 \%$; however, with the greatest clarity in band structure and highest amount of aggregation near the gel pockets.

Prominent bands of each flattened conformation were excised from the gel, and structures were isolated via spin filtration before being imaged by TEM and AFM, as shown in Figure 3. In each case, most structures were found in the configuration suggested by the pattern of openings along the struts. Vertices forming connections between three partial or complete struts appeared to be well formed, and the length of spanning segments was in the range of the ideally expected $75 \mathrm{~nm}$. In some cases, partial bundles resulting from an intentionally opened gap were not clearly distinguishable, likely due to the small size of these parts and possible overlapping with other struts. Populations containing fewer deformed or aggregated objects were generally seen under observation via AFM, likely resulting from the lack of potentially harsh drying steps which are typical in uranyl acetate negative staining used for TEM observations.

Taken in concert, the different two-dimensional frames demonstrate, on an individual basis, the ability for each vertex and strut, whether continuous or containing a gap region, to correctly anneal as a part of a particular flat variant of the tetrahedron. This does not fully eliminate the possibility that unintended internal stresses resulting from closure of the full structure lead to some degree of deformation. However, the single primary band migrating with the speed different to those of all open conformations 


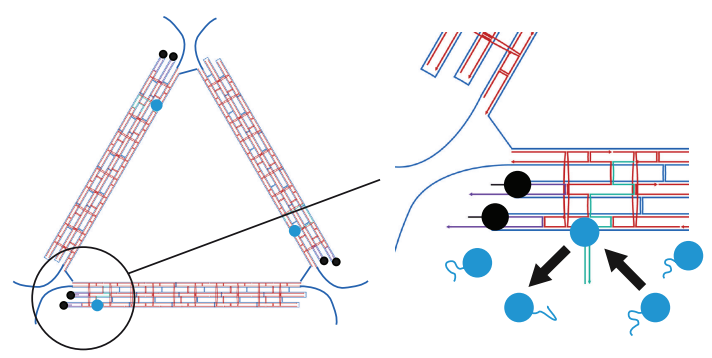

(a)

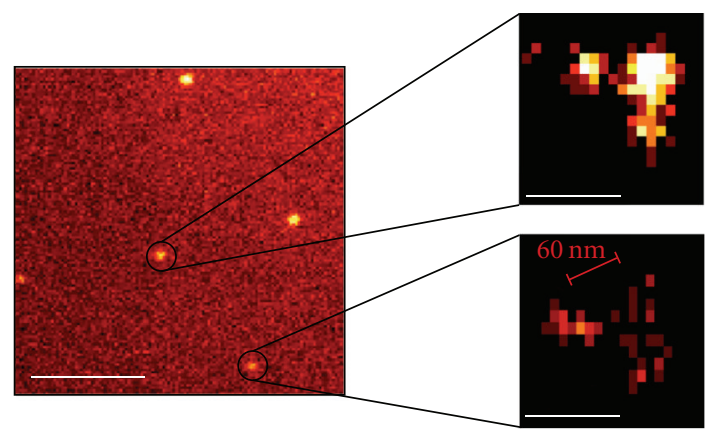

(b)

FIgure 4: DNA PAINT measurements on the triangular base of the tetrahedron. (a) Design schematic of the base showing docking sites for complimentary biotin- and ATTO655-conjugated oligonucleotides. The scaffold path is shown in blue, and staples lacking extended docking sequences are in red. Only basal struts 1,2 , and 3 are shown in the full schematic (left), and local detail at docking sites on strut 1 is given (right). The six extended staples containing docking sites for complimentary biotin-nucleotides are shown in purple, with biotin and the affixed nucleotide strand in black. Likewise, the three docking staples for ATTO655 dye are shown in green, while the ATTO655 dye and attached 8-base nucleotide which transiently hybridize to the structures are in light blue. (b) Resolution of triangular structure via DNA PAINT. (Left) Sample frame and (right) superresolution 2D reconstruction of local binding events. In the color scheme on the reconstructions, lighter colors correspond to a higher number of binding events, showing the underlying triangular form matching the schematic arrangement of dyes. For a comparison, the approximate expected distance between docking sites of $60 \mathrm{~nm}$ is indicated in the image. Scale bars: $4 \mu \mathrm{m}$ (left) and $100 \mathrm{~nm}$ (right).

is strong evidence for the successful annealing of the full tetrahedron. From this, we can conclude that rupturing occurs during fixing and staining procedures onto carboncoated TEM grids due to strong surface interactions from surface adsorption or transverse shear forces incurred in washing and drying steps.

3.4. Superresolution Analysis via DNA PAINT. Single-layer DNA origami structures have recently been shown to function as ideal substrates for superresolution microscopy techniques such as DNA PAINT [24] (points accumulation for imaging in nanoscale topography) or Blink microscopy [19] due to the ability to place fluorescent dyes at prescribed locations on the structure's surface with nanometer precision. The localization of fluorescent molecules beyond the normal diffraction limit of visible light is predicated by the principle that only a single molecule within the detection area is active, that is, emitting fluorescent light, at any given time $[22,23]$. Switching between ON and OFF states of fluorescent molecules is generally controlled by either chemical means or by utilizing intrinsic dark states [33]. In contrast, for systems involving interactions on surfacebound DNA-based nanostructures due to hybridizationbased binding and unbinding between dye-oligonucleotideconjugate detector strands and complimentary docking strands on the structures, switching occurs as a natural consequence of two factors: (i) freely diffusing strands within solution cause minimal background due to TIR excitement of only dyes near to the surface by the evanescent wave and (ii) a $70 \%$ increase of fluorescence is attained upon hybridization due to a significant reduction of intrastrand guanine quenching [24]. This results in the relatively straightforward detection of single hybridization events occurring between single, labeled detector oligonucleotides and complimentary docking strands extending outward from the surface of the origami object. Furthermore, control over rates of binding/unbinding events can be achieved by altering the length of the hybridized segment and concentration of the detector strand within the surrounding solution, respectively [24].

Binding of tetrahedra to a streptavidin-coated surface was accomplished by the inclusion of six handle sites for complimentary biotin-oligonucleotide hybridization, two each extending from the ends of struts 1,2 , and 3, as indicated in Figure 4(a) and in Figure S1 of the Supplementary materials. Passivation of the glass surface with a protein layer containing BSA and streptavidin has the additional advantage of shielding the negatively charged structures from the strong electrostatic binding which proves destructive to their three-dimensional morphology as seen with the carbon or mica surfaces described in the previous sections. In addition, one staple with an extended handle sequence extending from the $3^{\prime}$ end for ATTO655-oligonucleotide docking was incorporated into each of struts 1, 2, and 3, as outlined in detail for strut 1 in Figure 4(a) and shown for all struts in the Supplementary materials in Figure S1. The positioning of each dye site along its respective strut is shown in Figure 4(a), which together form a triangular arrangement with approximately $60 \mathrm{~nm}$ dimension. An 8base docking length for the detector oligonucleotide was selected to give sufficiently long binding times for accurate position determination, and a high detector concentration of $50 \mathrm{nM}$ was chosen in order to have high enough association rates [24].

Binding/unbinding events were recorded over $100 \mathrm{~s}$ with a capture rate of $20 \mathrm{~Hz}$ during TIR excitation from a singlemode diode laser with wavelength of $650 \mathrm{~nm}$. Recorded movies were analyzed in a custom software package programmed in LabVIEW [34]. Spots where binding events in each frame occurred were fitted with a 2D Gaussian, and the peak coordinates of each spot were entered into a $2 \mathrm{D}$ histogram with a binning of $10 \mathrm{~nm}$. According to the color scheme used, brighter spots represent a greater frequency of binding events at that particular position. 
The left frame in Figure 4(b) shows a single snapshot of the $11.5 \mu \mathrm{m} \times 11.5 \mu \mathrm{m}$ area monitored, and the two frames on the right show the superresolved 2D histograms of two different localized areas of binding/unbinding events. In contrast to raw data, the superresolved histograms of local spots indicate a localization of three peak event occurrences. The three apparent binding locations on the origami object display the correct distance scale and geometrical arrangement for spacing suggested by docking site placement along the base of the tetrahedron. Through the analysis of six docking site pairs, we found an average distance between localized histogram peaks of $(68 \pm 12) \mathrm{nm}$. For a comparison, the approximate expected distance of $60 \mathrm{~nm}$ is indicated in the image. Our data generally demonstrates the ability for DNA PAINT to resolve subdiffraction limit structures in two dimensions as well as the potential of such a DNA origami framework for superresolution techniques.

\section{Conclusion}

The construction of wire-frame, cage-like structures of nanometer size is an endeavor which not only holds great potential for studies requiring mechanically stable nanoscale spacers and scaffoldings but also could prove crucial for the development of containers suitable for targeted drug delivery. In this work, we have presented a DNA origamibased design for the simplest architecture of this kind; a fourfaced tetrahedron consisting of six flexibly jointed struts. Analysis of annealed objects via gel electrophoresis strongly indicates the presence of a population of uniformly annealed tetrahedra comprising approximately $20 \%$ of the total number of scaffolded objects, although structures appear to suffer ruptures at select weakened points along the struts likely due to forces occurring during electrostatic adsorption to surfaces and staining procedures. Intentional, targeted opening of these weak "gaps" in the struts via selected staple oligonucleotide omission led to the formation of a set of four different open configurations, again at a yield of approximately $20 \%$, which were analyzed by gel electrophoresis and visualized via atomic force microscopy and transmission electron microscopy. We found populations of structures that displayed the expected open morphologies when bound to surfaces, which demonstrated proper assembly of the individual vertices and full struts, further supporting the suggestion of rupture of full three-dimensional frameworks from external forces. By exploiting the nanometerprecise positioning of dyes on the tetrahedra, a key feature provided by the DNA origami technique, its triangular base was used as a stage for visualization in two dimensions with the superresolution DNA PAINT microscopy method. Analysis confirmed the triangular arrangement of attached dyes and similar spacing as predicted by the structural schematic. As a consequence, we expect such structures to be suitable test objects for $3 \mathrm{D}$ superresolution microscopy.

While the observed breakage of the tetrahedra at weakened points along the struts is proposed to arise from external stresses during fixation and staining, this apparent structural instability under stress is nevertheless a concern for future implementation of such objects as stable loadbearing scaffolds or cargo-bearing containers. This can potentially be resolved by longer overlaps between the connecting staples spanning the individual helices or by the incorporation of a linear scaffold strand into future designs to reduce the weakened gap regions along the struts imposed by the necessity of maintaining circular scaffold continuity. Enzymatic ligation of abutting $3^{\prime}$ and $5^{\prime}$ ends of staples within origami structures has been posited as a potential means to increase the overall stability of singlelayer architectures [35] and could serve to further strengthen three-dimensional frameworks as presented in this work. Additionally, recently developed techniques for metallization of DNA nanostructures could be applied as a potential means to mechanically stabilize and electrically functionalize the tetrahedron and its two-dimensional variants [36-38].

\section{Acknowledgments}

This work was financially supported by funding from NIM, CeNS, and DFG (LI1743/2-1, TI329/5-1). The authors would like to additionally acknowledge Dr. Susanne Kempter for assistance with transmission electron microscopy imaging and both Philipp Nickels and Dr. Ralf Jungmann for their help with atomic force microscopy visualization.

\section{References}

[1] N. C. Seeman, "Nucleic acid junctions and lattices," Journal of Theoretical Biology, vol. 99, no. 2, pp. 237-247, 1982.

[2] E. Winfree, F. Liu, L. A. Wenzler, and N. C. Seeman, "Design and self-assembly of two-dimensional DNA crystals," Nature, vol. 394, no. 6693, pp. 539-544, 1998.

[3] J. Malo, J. C. Mitchell, C. Vénien-Bryan et al., "Engineering a 2D protein-DNA crystal," Angewandte Chemie-International Edition, vol. 44, no. 20, pp. 3057-3061, 2005.

[4] Y. He, Y. Chen, H. Liu, A. E. Ribbe, and C. Mao, "Self-assembly of hexagonal DNA two-dimensional (2D) arrays," Journal of the American Chemical Society, vol. 127, no. 35, pp. 1220212203, 2005.

[5] J. Zheng, J. J. Birktoft, Y. Chen et al., "From molecular to macroscopic via the rational design of a self-assembled 3D DNA crystal,” Nature, vol. 461, no. 7260, pp. 74-77, 2009.

[6] J. Chen and N. C. Seeman, "Synthesis from DNA of a molecule with the connectivity of a cube," Nature, vol. 350, no. 6319, pp. 631-633, 1991.

[7] Y. Zhang and N. C. Seeman, "Construction of a DNAtruncated octahedron," Journal of the American Chemical Society, vol. 116, no. 5, pp. 1661-1669, 1994.

[8] W. M. Shih, J. D. Quispe, and G. F. Joyce, "A 1.7-kilobase single-stranded DNA that folds into a nanoscale octahedron," Nature, vol. 427, no. 6975, pp. 618-621, 2004.

[9] J. Zimmermann, M. P. J. Cebulla, S. Mönninghoff, and G. von Kiedrowski, "Self-assembly of a DNA dodecahedron from 20 trisoligonucleotides with $\mathrm{C}_{3 h}$ linkers," Angewandte ChemieInternational Edition, vol. 47, no. 19, pp. 3626-3630, 2008.

[10] R. P. Goodman, I. A. T. Schaap, C. F. Tardin et al., "Chemistry: rapid chiral assembly of rigid DNA building blocks for molecular nanofabrication," Science, vol. 310, no. 5754, pp. 1661-1665, 2005. 
[11] Z. Li, B. Wei, J. Nangreave et al., "A replicable tetrahedral nanostructure self-assembled from a single DNA strand," Journal of the American Chemical Society, vol. 131, no. 36, pp. 13093-13098, 2009.

[12] Y. He, T. Ye, M. Su et al., "Hierarchical self-assembly of DNA into symmetric supramolecular polyhedra," Nature, vol. 452, no. 7184, pp. 198-201, 2008.

[13] P. W. K. Rothemund, "Folding DNA to create nanoscale shapes and patterns," Nature, vol. 440, no. 7082, pp. 297-302, 2006.

[14] S. M. Douglas, H. Dietz, T. Liedl, B. Högberg, F. Graf, and W. M. Shih, "Self-assembly of DNA into nanoscale threedimensional shapes," Nature, vol. 459, no. 7245, pp. 414-418, 2009.

[15] B. Högberg, T. Liedl, and W. M. Shih, "Folding DNA origami from a double-stranded source of scaffold," Journal of the American Chemical Society, vol. 131, no. 26, pp. 9154-9155, 2009.

[16] E. Pound, J. R. Ashton, H. A. Becerril, and A. T. Woolley, "Polymerase chain reaction based scaffold preparation for the production of thin, branched DNA origami nanostructures of arbitrary sizes," Nano Letters, vol. 9, no. 12, pp. 4302-4305, 2009.

[17] S. M. Douglas, J. J. Chou, and W. M. Shih, "DNA-nanotubeinduced alignment of membrane proteins for NMR structure determination," Proceedings of the National Academy of Sciences of the United States of America, vol. 104, no. 16, pp. 66446648, 2007.

[18] I. H. Stein, V. Schüller, P. Böhm, P. Tinnefeld, and T. Liedl, "Single-molecule FRET ruler based on rigid DNA origami blocks," ChemPhysChem, vol. 12, no. 3, pp. 689-695, 2011.

[19] C. Steinhauer, R. Jungmann, T. L. Sobey, F. C. Simmel, and P. Tinnefeld, "DNA origami as a nanoscopic ruler for superresolution microscopy," Angewandte Chemie-International Edition, vol. 48, no. 47, pp. 8870-8873, 2009.

[20] T. Liedl, B. Högberg, J. Tytell, D. E. Ingber, and W. M. Shih, "Self-assembly of three-dimensional prestressed tensegrity structures from DNA," Nature Nanotechnology, vol. 5, no. 7, pp. 520-524, 2010.

[21] H. Dietz, S. M. Douglas, and W. M. Shih, "Folding DNA into twisted and curved nanoscale shapes," Science, vol. 325, no. 5941, pp. 725-730, 2009.

[22] B. Huang, M. Bates, and X. Zhuang, "Super-resolution fluorescence microscopy," Annual Review of Biochemistry, vol. 78, pp. 993-1016, 2009.

[23] S. W. Hell, "Microscopy and its focal switch," Nature Methods, vol. 6, no. 1, pp. 24-32, 2009.

[24] R. Jungmann, C. Steinhauer, M. Scheible, A. Kuzyk, P. Tinnefeld, and F. C. Simmel, "Single-molecule kinetics and superresolution microscopy by fluorescence imaging of transient binding on DNA origami," Nano Letters, vol. 10, no. 11, pp. 4756-4761, 2010.

[25] S. M. Douglas, A. H. Marblestone, S. Teerapittayanon, A. Vazquez, G. M. Church, and W. M. Shih, "Rapid prototyping of 3D DNA-origami shapes with caDNAno," Nucleic Acids Research, vol. 37, no. 15, pp. 5001-5006, 2009.

[26] R. Jungmann, T. Liedl, T. L. Sobey, W. Shih, and F. C. Simmel, "Isothermal assembly of DNA origami structures using denaturing agents," Journal of the American Chemical Society, vol. 130, no. 31, pp. 10062-10063, 2008.

[27] C. E. Castro, F. Kilchherr, D.-N. Kim et al., "A primer to scaffolded DNA origami," Nature Methods, vol. 8, no. 3, pp. 221-229, 2011.

[28] A. Rajendran, M. Endo, Y. Katsuda, K. Hidaka, and H. Sugiyama, "Programmed two-dimensional self-assembly of multiple DNA origami jigsaw pieces," ACS Nano, vol. 5, no. 1, pp. 665-671, 2011.

[29] K. N. Kim, K. Sarveswaran, L. Mark, and M. Lieberman, "Comparison of methods for orienting and aligning DNA origami," Soft Matter, vol. 7, no. 10, pp. 4636-4643, 2011.

[30] M. Rief, H. Clausen-Schaumann, and H. E. Gaub, "Sequencedependent mechanics of single DNA molecules," Nature Structural Biology, vol. 6, no. 4, pp. 346-349, 1999.

[31] T. Strunz, K. Oroszlan, R. Schäfer, and H. J. Güntherodt, "Dynamic force spectroscopy of single DNA molecules," Proceedings of the National Academy of Sciences of the United States of America, vol. 96, no. 20, pp. 11277-11282, 1999.

[32] M. Grandbois, M. Beyer, M. Rief, H. Clausen-Schaumann, and H. E. Gaub, "How strong is a covalent bond?" Science, vol. 283, no. 5408, pp. 1727-1730, 1999.

[33] C. Steinhauer, C. Forthmann, J. Vogelsang, and P. Tinnefeld, "Superresolution microscopy on the basis of engineered dark states," Journal of the American Chemical Society, vol. 130, no. 50, pp. 16840-16841, 2008.

[34] Software for PAINT analysis, http://www.e14.ph.tum.de.

[35] A.-P. Eskelinen, A. Kuzyk, T. K. Kaltiaisenaho et al., "Assembly of single-walled carbon nanotubes on DNA-origami templates through streptavidin-biotin interaction," Small, vol. 7, no. 6, pp. 746-750, 2011.

[36] R. Schreiber, S. Kempter, S. Holler et al., "DNA origami templated growth of arbitrarily shaped metal nanoparticles," Small, vol. 7, no. 13, pp. 1795-1799, 2011.

[37] K. Keren, M. Krueger, R. Gilad, G. Ben-Yoseph, U. Sivan, and E. Braun, "Sequence-specific molecular lithography on single DNA molecules," Science, vol. 297, no. 5578, pp. 72-75, 2002.

[38] J. Liu, Y. Geng, E. Pound et al., "Metallization of branched DNA origami for nanoelectronic circuit fabrication," ACS Nano, vol. 5, no. 3, pp. 2240-2247, 2011. 

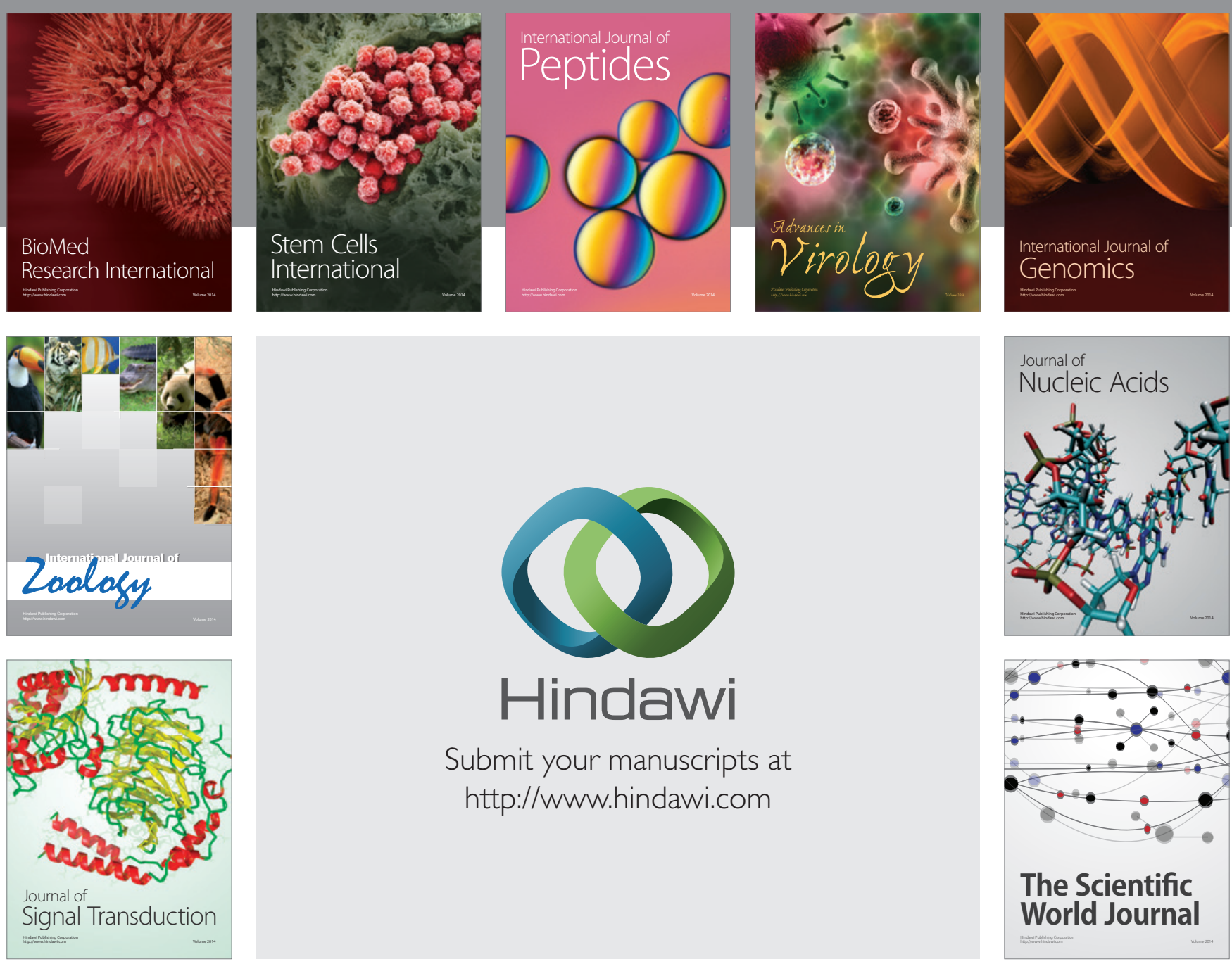

Submit your manuscripts at

http://www.hindawi.com
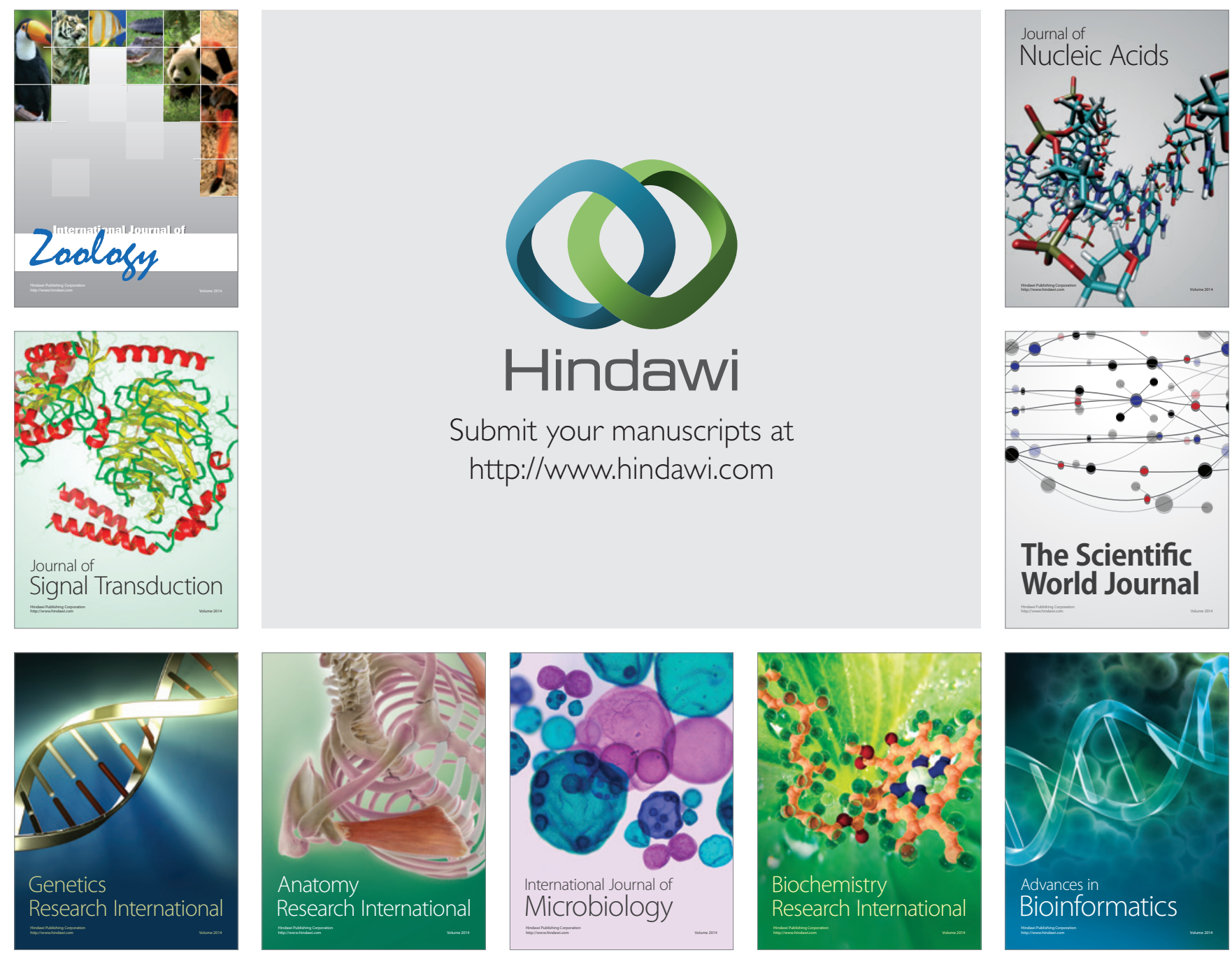

The Scientific World Journal
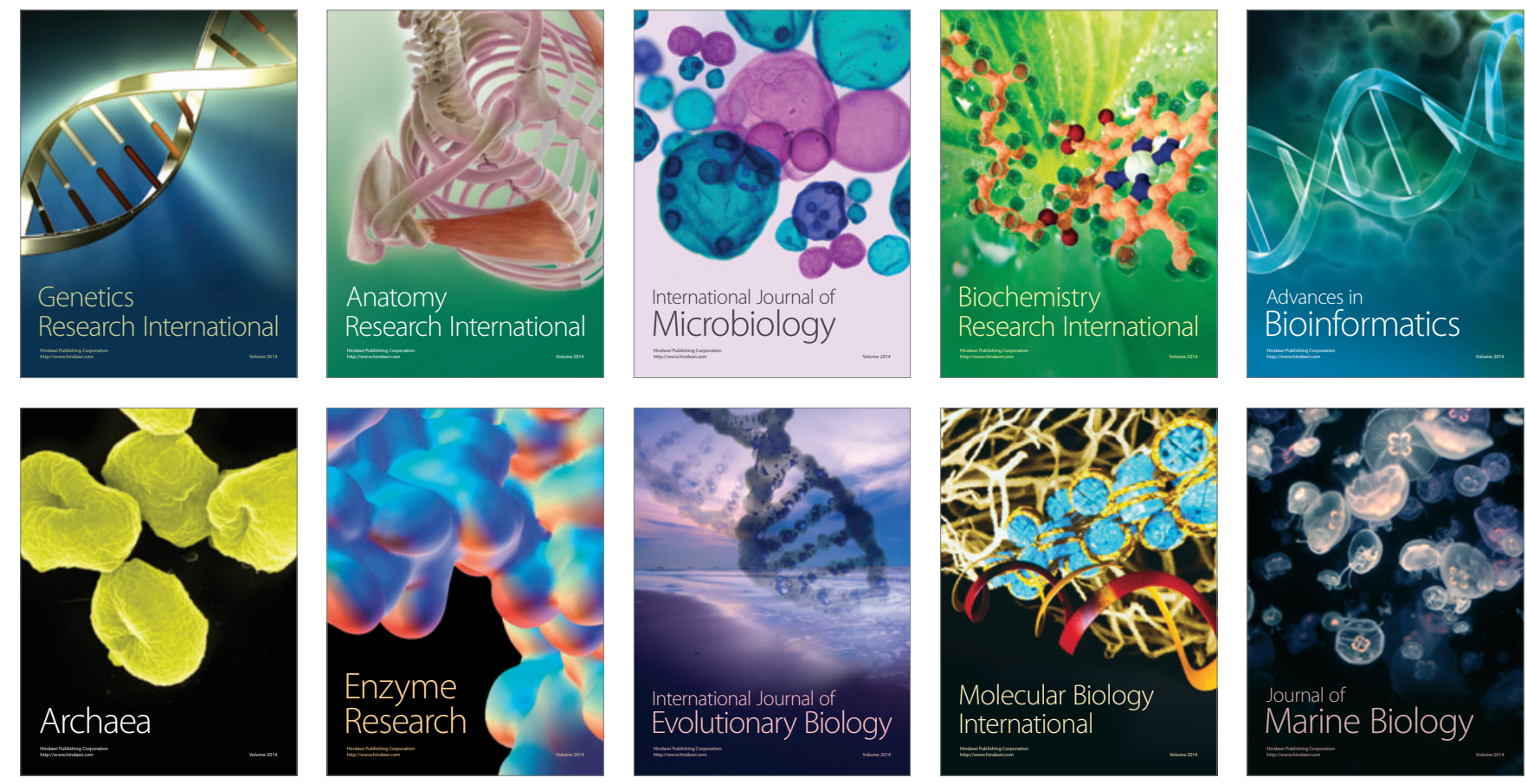